\title{
Electric field effect on superconductivity in $\mathrm{La}_{2-x} \mathrm{Sr}_{x} \mathrm{CuO}_{4}$
}

\author{
G. Dubuis,,$^{1,2}$ A. T. Bollinger, ${ }^{1}$ D. Pavuna, ${ }^{2}$ and I. Božović ${ }^{1}$ \\ ${ }_{1}^{1}$ Brookhaven National Laboratory, Upton, New York 11973-5000, USA \\ ${ }^{2}$ Ecole Polytechnique Fédérale de Lausanne, CH-1015 Lausanne, Switzerland
}

(Received 15 April 2011; accepted 29 July 2011; published online 15 June 2012)

\begin{abstract}
We demonstrate a method to tune the carrier concentration of a high temperature superconductor over a wide range, using an applied electric field. Thin film devices were made in an electrical double layer transistor configuration utilizing an ionic liquid. In this way, the surface carrier density in $\mathrm{La}_{2-x} \mathrm{Sr}_{x} \mathrm{CuO}_{4}$ films can be varied between 0.01 and 0.14 carriers per $\mathrm{Cu}$ atom with a resulting change in critical temperature of $25 \mathrm{~K}(\sim 70 \%$ of the maximum critical temperature in this compound). This allows one to study a large segment of the cuprate phase diagram without altering the level of disorder. We used this method [A. T. Bollinger et al., Nature 472, 458-460 (2011)] to study the quantum critical point at the superconductor to insulator phase transition on the underdoped side of superconducting dome, and concluded that this transition is driven by quantum phase fluctuations and Cooper pair delocalization. (C) 2012 American Institute of Physics.

[http://dx.doi.org/10.1063/1.4726158]
\end{abstract}

\section{INTRODUCTION}

As the concentration of dopant atoms is increased, the ground state behavior of cuprate compounds changes dramatically, from antiferromagnetic insulator, to a hightemperature superconductor (HTS) to a Fermi liquid metal. However, this usually involves substantial chemical modification, as well as increase in (dopant-induced) structural disorder. An attractive alternative, widely used in the semiconductor technology, is to alter the density of free charge carriers by applying an external electric field. However, attempts to fabricate superconducting field effect devices has been hampered by the large values of the carrier concentrations $\left(10^{21}-10^{22} \mathrm{~cm}^{-3}\right)$, which correspond to screening length less than $1 \mathrm{~nm}$.

The common field-effect transistor geometry uses a metallic top gate that is separated from the material to which field is applied by a gate insulator. The insulator must have a breakdown field that is larger than the electric fields to be applied and also should be chosen to minimize leakage currents. This geometry has been employed to study the field effect in cuprates ${ }^{2}$ but the limiting factor has been the breakdown field of the gate dielectric. To induce large changes in the carrier concentration, on the order of $10^{21} \mathrm{~cm}^{-3}$, in just a single unit cell (UC) thick layer, one would need at least $10^{8} \mathrm{~V} / \mathrm{cm}$, a field that no known insulator can withstand. In an early report of significant field modulation of superconductivity, Mannhart et al. ${ }^{3}$ utilized an inverted metal-insulator-superconductor geometry where the gate electrode was located on the bottom of the substrate, atop of which a $\mathrm{YBa}_{2} \mathrm{Cu}_{3} \mathrm{O}_{7-\delta}$ film was grown. By applying a voltage to the back gate, they were able to modify the carrier density in the film and shift the superconducting critical temperature, $T_{c}$, by several Kelvin. The same geometry has been used subsequently to achieve $10 \mathrm{~K}$ shifts in $T_{c}$ in $\mathrm{Y}_{1-y} \mathrm{Ca}_{y} \mathrm{Ba}_{2} \mathrm{Cu}_{3} \mathrm{O}_{7-\delta}$ films and $\mathrm{NdBa}_{2} \mathrm{Cu}_{3} \mathrm{O}_{7-\delta}$ films. ${ }^{4-6}$ In these studies, the cuprate films were grown on $\mathrm{SrTiO}_{3}$, which has a large dielectric constant; this increases the capacitance and helps achieve a large surface-charge density. Back gating $\mathrm{SrTiO}_{3}$ has also recently been employed to induce superconductivity, with $T_{c}=310 \mathrm{mK}$, in the two dimensional electron gas at the $\mathrm{LaAlO}_{3}-\mathrm{SrTiO}_{3}$ interface. $^{7}$

An alternative approach has been to use a ferroelectric such as $\mathrm{PbZr}_{x} \mathrm{Ti}_{1-x} \mathrm{O}_{3}$ as the gate insulator. An applied gate voltage is used to change the polarization of the ferroelectric, and its polarization field then is responsible for changing the carrier concentration in the HTS film. Once the ferroelectric is polarized, the gate voltage can be turned off so that in this scheme gate leakage currents can be eliminated. Modulation of the superconductivity in $\mathrm{GdBa}_{2} \mathrm{Cu}_{3} \mathrm{O}_{7-\delta}$ and $\mathrm{NdBa}_{2} \mathrm{Cu}_{3} \mathrm{O}_{7-\delta}$ has been observed in this way with $T_{c}$ shifts of several Kelvin. ${ }^{8,9}$

Yet another approach is to use solid or liquid electrolytes. Application of a gate voltage $\left(V_{G}\right)$ between the gate electrode and the film causes ions of the same polarity as $V_{G}$ to move to the film surface forming a Helmholtz electric double layer (EDL) at the electrolyte-film interface (Fig. 1(a)). This technique, inspired by electrolyte supercapacitors, ${ }^{10-12}$ is known to create quite high electric fields of the order $10^{8} \mathrm{~V} / \mathrm{cm}$. Electron accumulation using polymer electrolytes (a salt dissolved in a polymer such as polyethylene glycol) have resulted $^{10}$ in surface carrier densities as high as $10^{14} \mathrm{~cm}^{-2}$, and even higher density, $8 \times 10^{14} \mathrm{~cm}^{-2}$, has been achieved using an ionic liquid as the electrolyte. ${ }^{13}$ This technique has recently been employed to induce superconductivity in an insulator $\left(\mathrm{SrTiO}_{3}\right)$ as well as a semiconductor $(\mathrm{ZrNCl})^{10,11,14}$ It enabled us ${ }^{1}$ to induce a substantial modification of the carrier density of cuprates, and hence in this paper we adopt the electrolyte route to study the field effect in HTS materials.

In this paper, we show that in EDL structures fabricated from $\mathrm{La}_{2-x} \mathrm{Sr}_{x} \mathrm{CuO}_{4}$ (LSCO) thin films, superconductivity can be tuned over a wide range (with changes in $T_{c}$ up to $25 \mathrm{~K})$. Moreover, unlike chemical doping, the field-effect 
presumably just modifies the surface charge density, without changing the level of disorder.

\section{EXPERIMENT}

To synthesize the films, we use an advanced oxide atomic-layer-by-layer molecular beam epitaxy system. It is capable of reproducible fabrication of atomically smooth films of cuprates with control over the stoichiometry at the $1 \%$ level and digital control of layer thickness (Fig. 1(a)). ${ }^{15-18}$ Since the electrostatic screening length for cuprates is of the order of one unit cell, it is imperative that the deposited films are continuous and defect-free on the same length scale. The crystal structure of films was monitored in situ during growth by reflection high energy electron diffraction (RHEED) and after growth studied by x-ray diffraction. Both techniques as well as atomic force microscopy showed the films to be of high quality and without secondary-phase precipitates.

Using standard photolithography, ion milling, and e-beam evaporation techniques, the films were patterned into sets of rectangular bars of various aspect ratios. The widths of the rectangular devices were $10-500 \mu \mathrm{m}$ and the lengths were $500-900 \mu \mathrm{m}$, with multiple voltage contacts regularly

(a)

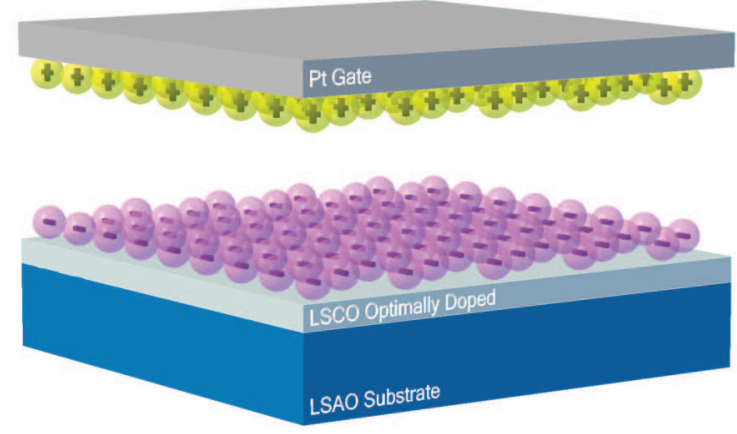

(c)

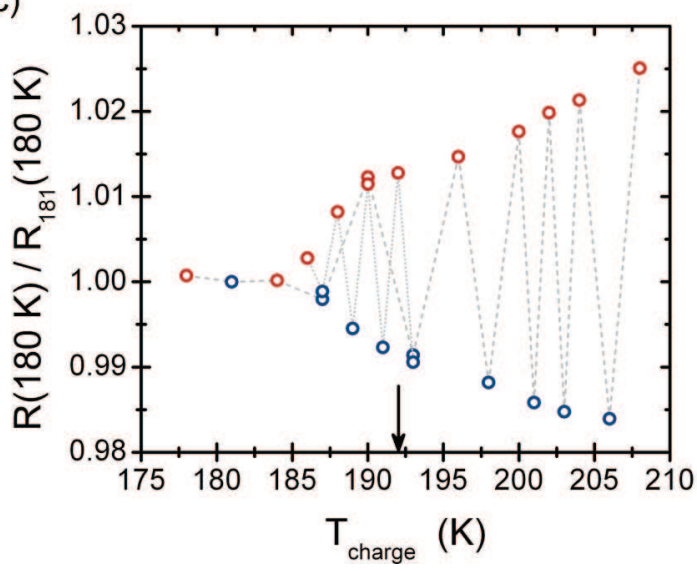

distributed on both sides of the strip, 50 to $300 \mu \mathrm{m}$ apart (Fig. 2). Different device patterns (see Fig. 2) were designed and utilized. Some of the wire bonds used to connect the device to the sample holder are clearly visible. The simplest devices were fabricated using our standard lithography masks and just adding a platinum foil top gate (Figs. 2(a) and 2(b)) about $0.1-0.3 \mathrm{~mm}$ above the sample surface. The ionic liquid was kept in place between the gate and the sample by the capillary forces. In Fig. 2(a), the visible discoloration around the gate shows the part of the sample wetted by the ionic liquid. For highly resistive samples, a different mask was used with inverted aspect ratios, the width of $1 \mathrm{~cm}$, and the length between $3 \mu \mathrm{m}$ and $1 \mathrm{~mm}$ (Fig. 2(b)). Parallel gold electrodes, separated by gaps of increasing width, were deposited subsequently. The electrodes can be covered by a layer of insulator $\left(\mathrm{AlO}_{3}\right)$, except for the part, not covered by ionic liquid, where the wire bonds are attached. This ensures that during charging, the electric double layer is mainly formed on top of the free surface of the sample, and not confined just to the gold contacts with a much larger surface. As for the previous pattern, a platinum top gate is added, and the ionic liquid, not visible here, is kept in place by the capillary forces. The patterns in Figs. 2(c) and 2(d), which include coplanar gates (the largest visible gold patterns), were (b)

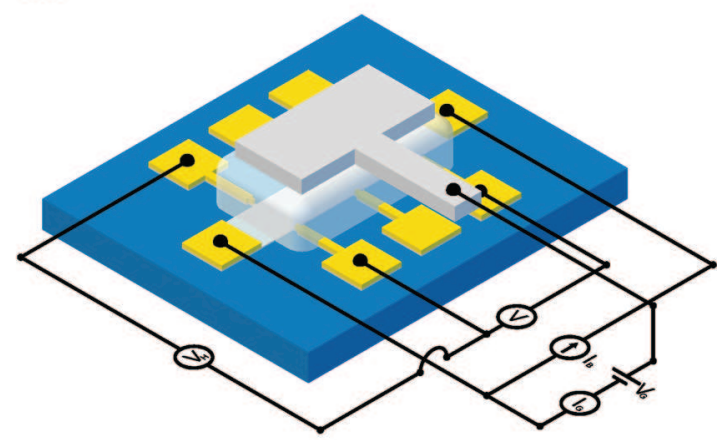

(d)

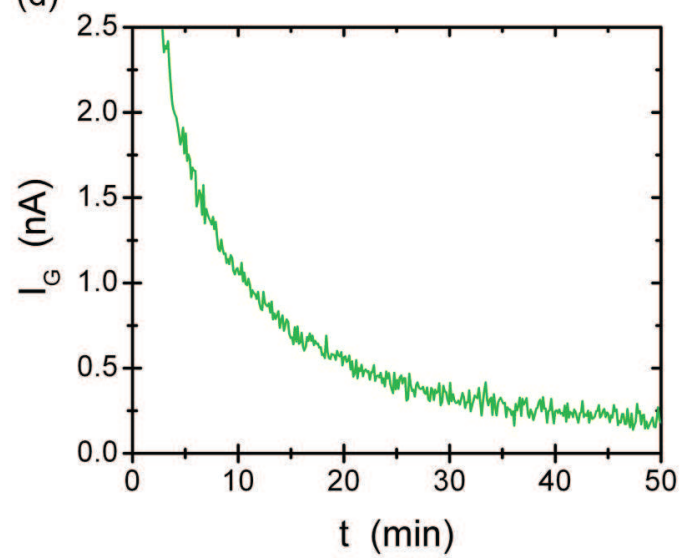

FIG. 1. (a) A diagram of the cuprate multilayer film used in this work. A negative gate voltage draws the cations (DEME ${ }^{+}$) to the platinum gate and the anions $\left(\mathrm{TFSI}^{-}\right.$) to the HTS film surface, respectively. (b) Schematic of the experimental setup. (c) The effect of charging at various charging temperatures, $T_{\text {charge }}$, with DEME-TFSI used as the gate electrolyte. (d) The gate current as a function of time for a representative charging cycle. 
(a)

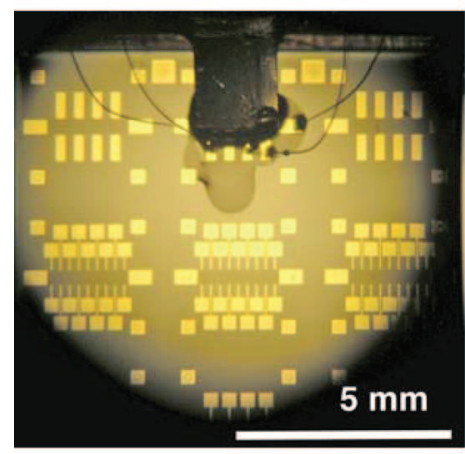

(c)

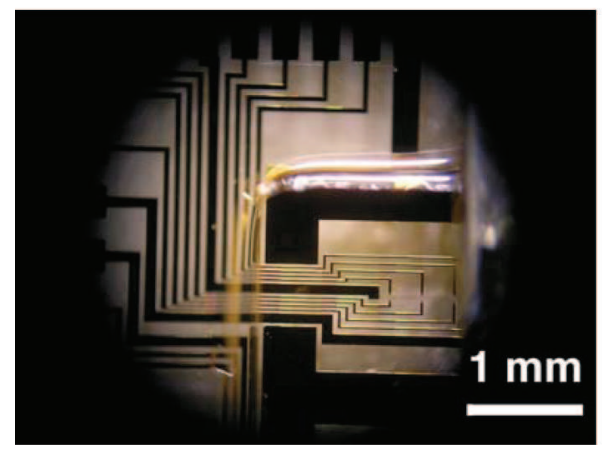

(b)

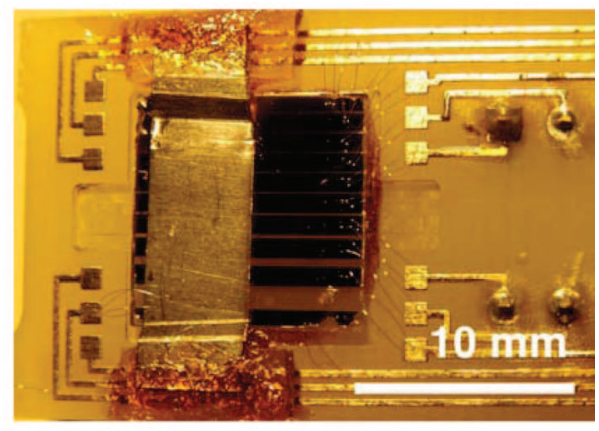

(d)

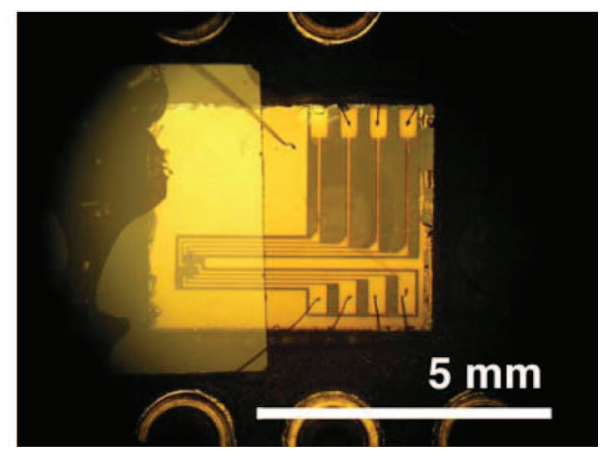

FIG. 2. Optical micrographs of various fabricated thin film devices used in this study; in all these samples, the ionic liquid used is DEME-TFSI. The resistance is measured using a 4-point configuration. (a) A simple 6-point contact strip pattern adapted to field effect studies. (b) A pattern used for highly resistive samples. (c) A pattern specifically designed in order to gate thin film samples with ionic liquid, with the devices surrounded by a larger coplanar gate. Only one out of six devices is visible in this picture. (d) Another pattern with four separate devices placed near to the center of $10 \times 10 \mathrm{~mm}^{2}$ film, designed to allow the sample to be diced. One of these four devices is mounted and wire-bonded; the gate is visible as the gold square at the top left of the sample. The glass cover slide is already in place, but the ionic liquid has not yet been deposited. designed specifically for gating with ionic liquid. Here, the ionic liquid is kept in place by the capillary force between the substrate surface and a microscope glass cover slide inserted just above the sample. The meniscus at the edge of the cover slide in Fig. 2(c) is clearly visible.

The rectangular devices were current biased with a bias current, $I_{B}$, between $10 \mathrm{nA}$ and $10 \mu \mathrm{A}$. A dual channel nanovoltmeter measured the longitudinal voltage, $V$, Delta mode measurements were used to eliminate offsets. Gate voltage was applied and gate current measured by an electrometer source-measure unit. Longitudinal resistance was measured first and then a gate electrolyte was applied between the device and the gate, either top or coplanar (Fig. 1(b)). Two different gate electrolytes were used in this work: (i) the ionic liquid N,N-diethyl-N-methyl-N-(2-methoxyethyl) ammonium bis(trifluoromethylsulphonyl)imide (DEME-TFSI) and (ii) a solution of sodium fluoride salt dissolved in polyethylene glycol (PEG-NaF). Since many different devices were simultaneously fabricated from each grown film, for comparison we applied simultaneously both gate electrolytes to different devices on the same wafer. We have found no significant difference between the two; the carrier density that could be induced using PEG-NaF and DEME-TFSI was essentially the same. The main difference between these two gate electrolytes is the much lower melting point of DEMETFSI, $\sim 200 \mathrm{~K}$ instead of $300 \mathrm{~K}$ for PEG, which allows for a faster cycling as well as charging the devices at a lower temperature and for a shorter time (Figs. 3(a) and 3(b)).

The amount of accumulated charges depends on $V_{G}$ as well as the charging time ( $\left.t_{\text {charge }}\right)$ and temperature $\left(T_{\text {charge }}\right)$. Temperature-dependant measurements were made during sample cooling while the applied $V_{G}$ was kept constant. To switch to a different gate voltage, $V_{G}$ was kept at its latest value and then the sample was heated to $T_{\text {charge }}$ where the new gate voltage was applied. For PEG-NaF, we used $T_{\text {charge }}=302 \mathrm{~K}$, whereas for DEME-TFSI we chose $T_{\text {charge }}=192-210 \mathrm{~K}$. For DEME-TFSI, $192 \mathrm{~K}$ is near the lowest temperature at which a discernable change due to charging could be detected in the resistance of the device (Fig. 1(c)). To determine this, the gate voltage was applied at various $T_{\text {charge }}$ and then $R(T)$ curves were measured. The gate was alternated between $+0.5 \mathrm{~V}$ and $-0.5 \mathrm{~V}$ from measurement to measurement. In Fig. 1(c), we plot for each charging cycle the resistance measured at $180 \mathrm{~K}$ after charging for the same fixed time $\left(30^{\prime}\right)$ but at different $T_{\text {charge }}$. This shows that the device properties could be changed by applying a gate voltage at $T_{\text {charge }}$ as low as $\sim 185 \mathrm{~K}$. Below this, temperature charging has no effect as the ions are frozen in place. This low temperature of charging allows us to extend the gate voltage range of DEME-TFSI to at least $\pm 20 \mathrm{~V}$ without damaging the sample, while still being able to induce changes in the charge of the EDL and thus change the behavior of the devices nondestructively. The usual charging time was $30 \mathrm{~min}$, which was sufficient for the ion motion in the electrolytes to slow down and a new charge state to be established (Fig. 1(d)). This relatively high charging voltage indicated that a significant loss of voltage occurs due to the high resistance of the ionic liquid at this low temperature. Note that the ionic liquid undergoes glassy transition to a solid and thus the mobility of the ions very gradually increases with temperature around the melting point. 


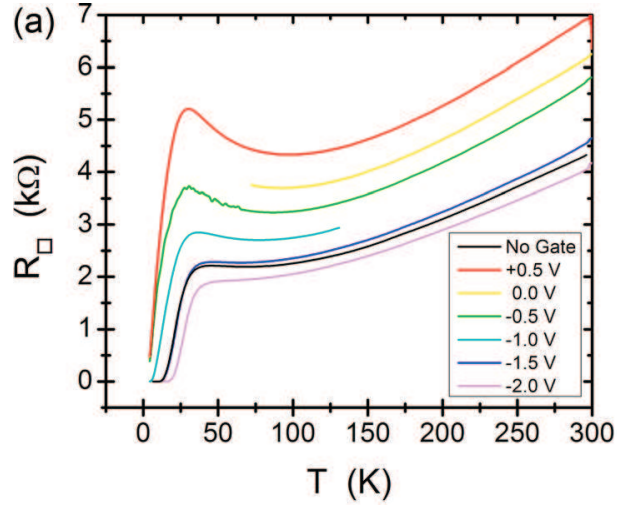

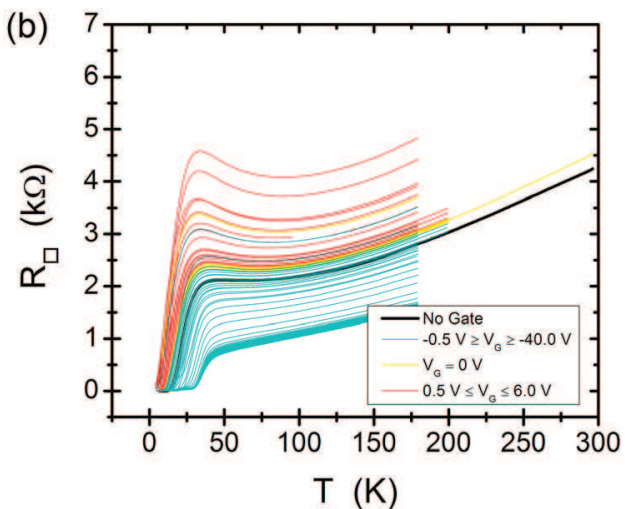

FIG. 3. The field effect in a sample composed of a 1 UC thick metallic $\mathrm{La}_{1.56} \mathrm{Sr}_{0.44} \mathrm{CuO}_{4}$ buffer, 5 UC thick insulating $\mathrm{La}_{2} \mathrm{CuO}_{4}$ layer, and $2 \mathrm{UC}$ thick $\mathrm{La}_{1.84} \mathrm{Sr}_{0.16} \mathrm{CuO}_{4}$. (a) Gating with PEGNaF. (b) Gating with DEME-TFSI.
After the first sets of measurement where we charged the sample at $192 \mathrm{~K}$, we tried various higher temperatures. Charging the sample at a higher temperature allowed us to reduce the charging time while getting a similar change in carrier concentration in the sample, with a reduced gate voltage. Systematic tests indicated that the optimal charging temperature is $T_{\text {charge }}=210 \pm 0.3 \mathrm{~K}$. At this higher charging temperature, the range of permissible gate voltages was much reduced, with irreversible damage occurring in some samples at gate voltages exceeding 3-4 V, depending on the particular sample. Attempts to further increase the charging temperatures (to above $220 \mathrm{~K}$ ) resulted in frequent damage to the gold contacts of the sample for $V_{G}>3 \mathrm{~V}$ and thus to losing the devices.

(a)

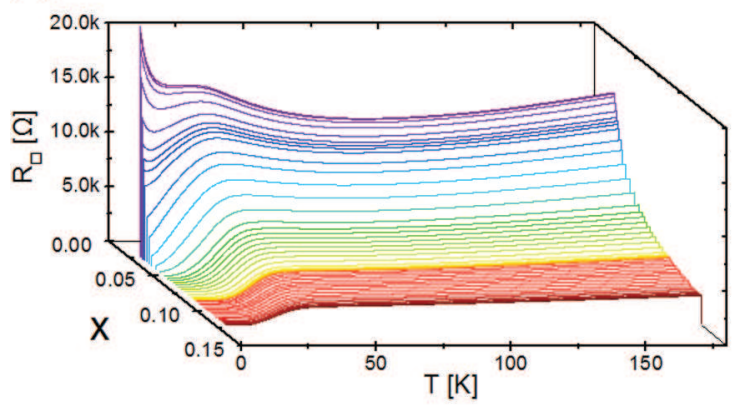

(c)

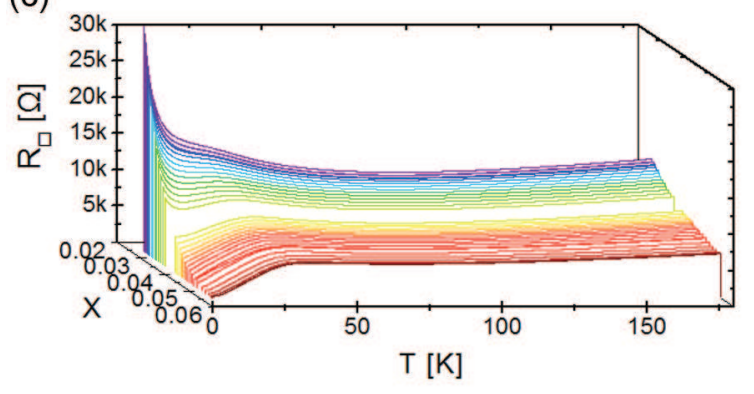

\section{RESULTS AND DISCUSSION}

In Figs. 4 and 5, we present measurements on three devices, fabricated from a single film of 3 UC thick $\mathrm{La}_{1.84} \mathrm{Sr}_{0.16} \mathrm{CuO}_{4}$ film (Fig. 4). The resistance vs. temperature, $R(T)$, dependence of the sample appeared slightly underdoped, i.e., they showed a local maximum on the $R(T)$ curve just before the superconducting transition. For all three devices, application of negative gate voltages, which corresponds to increase in hole density, lead to a decrease in resistance as well as a shift in $T_{c}$ to higher values; both findings are consistent with the hole-doped nature of LSCO. The normal state resistance at $180 \mathrm{~K}$ (below the freezing point of DEME-TFSI) was tunable by a factor of 5 to 15 .

(b)

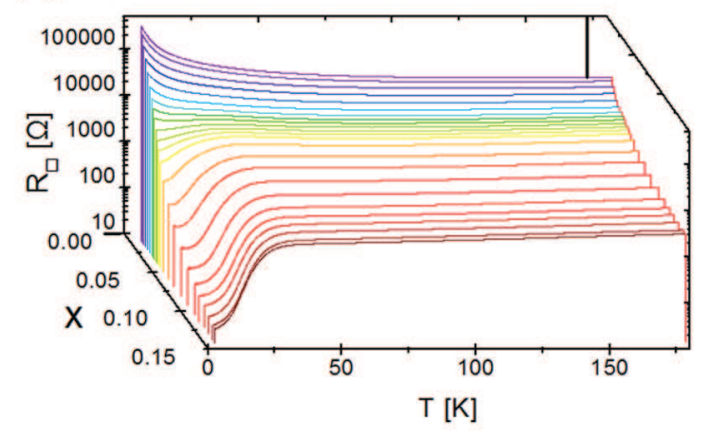

(d)

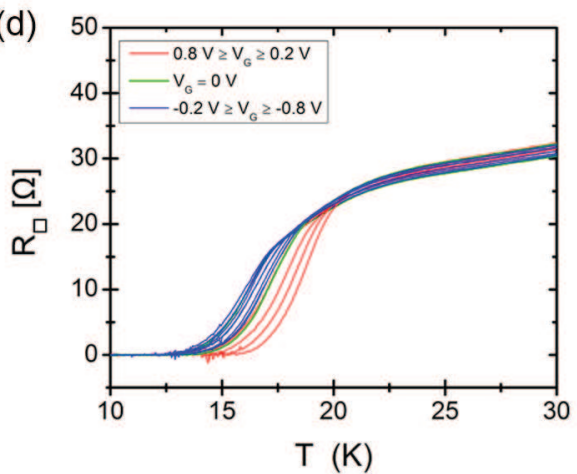

FIG. 4. Temperature dependence of resistance for superconducting field effect devices. Three similar devices ( $300 \mu \mathrm{m}$ long and $100 \mu \mathrm{m}$ wide) were patterned in a single film of $\mathrm{La}_{1.84} \mathrm{Sr}_{0.16} \mathrm{CuO}_{4}$ (optimally doped), 3 unit cells thick $\left(6 \mathrm{CuO}_{2}\right.$ layers), deposited on LaSrAlO . (a) Device 1 shows a complete range of effective carrier concentration, from $x=0.02$ to 0.12 . (b) Device 2 shows a range of carrier concentration from $x=0.009$ to 0.142 . This device could be driven deeper into the insulating state, thus, this graph is shown on a logarithmic scale. (c) Device 3 shows a range of carrier concentration from $x=0.025$ to 0.054 . But in this case the sample is not able to reach zero resistance above $4 \mathrm{~K}$ (d) An overdoped sample composed of 20 unit cells of metallic buffer and 2 unit cells of LSCO nominally doped with $x=0.18$. 


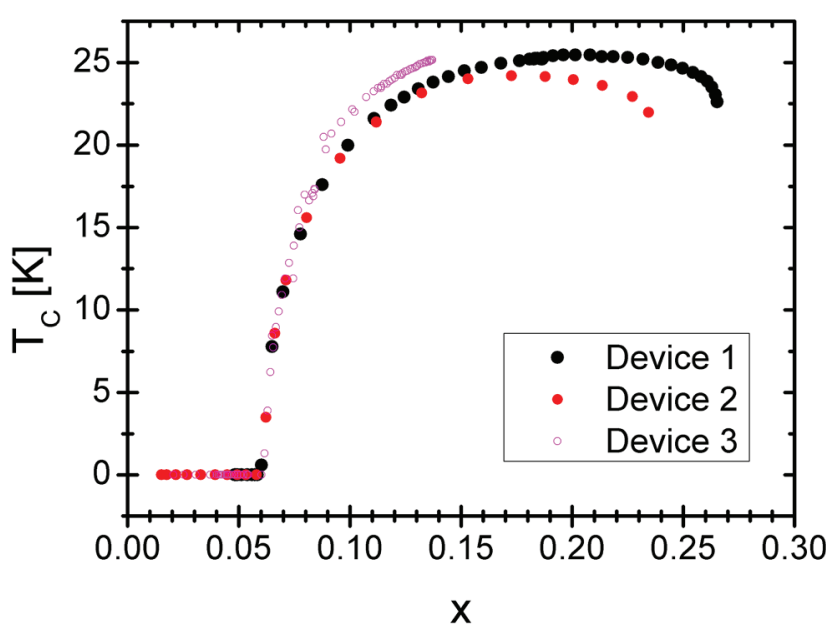

FIG. 5. Dependence of the critical temperature on the electric fieldcontrolled carrier concentration in three EDL devices with ionic liquid. Since these devices showed broad superconducting transitions, $T_{\mathrm{c}}$ is defined here as the midpoint of the transition. The surface charge density is only estimated and has been scaled to the onset of the dome at $\mathrm{x}=0.06$. A single EDL device can be used to probe the physics of a significant part of the phase diagram.

Large shifts in $T_{c}$, by more than $20 \mathrm{~K}$, were observed in all three devices. For example, in device 1, the mid-point of the resistive transition was tunable between $0 \mathrm{~K}$ and $25 \mathrm{~K}$. The known cuprate phase diagram shows that upon chemical doping on the underdoped side of the superconducting dome $T_{c}$ initially rises from zero steeply with doping and then gradually flattens as the optimal doping is reached. This is nicely reproduced in our field-doping experiments as can be seen in Fig. 5. Currently, we are able to cover more than half of the superconducting dome. Note that at this point it is difficult to determine precisely the carrier concentration in the topmost layer, because on one hand the unknown contribution to conductivity of the other layers below can decrease the measured resistance, while on the other surface contamination due to exposure to atmosphere can reduce the effective cross-section and thus increase the resistance. However, qualitatively our tested devices seem to follow the expected behavior.

When working with electrolytes, one should be alert to the possibility of chemical modification of the film. Indeed, if dopant atoms were electrochemically implanted into the device, this could mimic some of the same results reported here. However, for a number of reasons, we believe that the observed modulation in carrier density and $T_{c}$ are primarily due to electrostatic effects rather than chemical doping. First, we have found that in thick samples no significant reduction of critical temperature is possible, implying that any possible intercalation in LSCO ought to be limited to the topmost layer. ${ }^{1}$ Next, we studied a number of test samples to delineate the safe limits of operating gate voltage within which the field-induced modulation is both reproducible and reversible. Once the devices are operated outside this safe electrochemical window, one could see some signs of damage in the transport measurements. The devices shown here were measured between 24 and 84 times, each time with a different gate voltage (within the safe range).
We have also experimented with LSCO films of various doping levels. In overdoped films, we observed that applying a negative gate voltage, i.e., increasing the hole concentration makes the film more conductive (reduces the normal-state resistance) but reduces $T_{c}$ (see Fig. 4(d)). Applying a positive gate voltage has the opposite effect: the film becomes more resistive while $T_{c}$ increases (here, by more than $5 \mathrm{~K}$ ). Note that at this case the near-surface layer affected by the electric field is shunted by a thick metallic buffer under it, which makes a quantitative estimate of the change in the surface carrier density difficult.

Finally, it is important to point out some inherent limitations of this technique. One is that it is inherently slow-this is not a way to fabricate three-terminal electronic devices. Next, these ionic liquids are good polar solvents, which puts some restriction on the materials to which ionic liquid gating can be applied. Further, the induced changes in the carrier concentration are limited to an extremely thin layer at the surface of the sample. The thickness of this active layer is determined by the Thomas-Fermi screening length, which along the c-axis in LSCO has been determined to be of $6 \AA$, ${ }^{17}$ i.e., about the thickness of $0.5 \mathrm{UC}$ (one molecular layer) of LSCO. Thus, essentially only the topmost $\mathrm{CuO}_{2}$ layer can be significantly modified and the effect should vanish in the bulk.

The results so far seem to reproduce the superconducting dome well studied in cuprates using chemical doping. While we have no reasons to expect any qualitative differences, it is now possible to cover a wide range of charge carrier concentration with a nearly arbitrary resolution. This allows one to study in detail what happens in the neighborhood of quantum critical points in the phase diagram. ${ }^{1}$ Note that in cuprates $T_{c}$ can be maximized by tuning the carrier density; the same is true in other tunable families of compounds. Hence, another anticipated application of this technique may stem from the ability to significantly change the carrier concentration, in particular in materials where chemical doping is not feasible. Very recently, superconductivity in $\mathrm{KTaO}_{3}$ with $T_{c}=0.3 \mathrm{~K}$ was discovered using this technique. ${ }^{19}$

\section{CONCLUSIONS}

We have developed a method to reversibly change the carrier concentration in LSCO ultrathin films. In the particular films shown here, we were able to vary the carrier concentration per $\mathrm{Cu}$ atom from 0.01 to 0.14 , and measure a change in $T_{c}$ (defined here as the transition midpoint) from $0 \mathrm{~K}$ to $25 \mathrm{~K}$. This technique can be used to study the quantum critical points, by allowing a scan in the horizontal (carrier concentration) dimension of the phase diagram. This technique is not limited to cuprates only, but can be applied to any material compatible with the ionic liquid used. As there are thousands of room temperature ionic liquids available, ${ }^{20}$ it should be possible to gate many materials. By comparing chemical doped films to electrostatically doped ones, one may be able to isolate the effects of change in concentration of charge carriers from the effects of chemical and structural disorder induced by chemical doping. The main limitation is that the sample has to be conductive enough in order to allow 
the mirror charges in the film to migrate and compensate the ionic charges in the liquid at the surface of the sample and form the EDL. The present technique may allow tuning the carrier density in new compounds where chemical doping was not possible.

\section{ACKNOWLEDGMENTS}

The work at BNL was supported by the U.S. Department of Energy, Basic Energy Sciences, Materials Sciences and Engineering Division. A.T.B. was supported by the U.S. DOE, Energy Frontier Research Center. D.P. and G.D. were supported by the Laboratory for Physics of Complex Matter (EPFL) and the Swiss National Science Foundation. We thank R. Adzic, J. Misewich, J. Yoon, A. Gozar, G. Logvenov, J. Pereiro, J. Sadovsky, and R. Sundling for their enlightening discussions and technical support.

${ }^{1}$ A. T. Bollinger, G. Dubuis, J. Yoon, D. Pavuna, J. Misewich, and I. Božović, Nature 472, 458-460 (2011).

${ }^{2}$ A. Cassinese, G. M. De Luca, A. Prigiobbo, M. Salluzzo, and R. Vaglio, Appl. Phys. Lett. 84, 3933-3935 (2004).

${ }^{3}$ J. Mannhart, J. G. Bednorz, K. A. Müller, and D. G. Schlom, Z. Phys. B: Condens. Matter 83, 307-311 (1991).

${ }^{4}$ G. Yu. Logvenov, A. Sawa, and C. W. Schneider, Appl. Phys. Lett. 83, 3528-3530 (2003).
${ }^{5}$ G. Yu. Logvenov, C. W. Schneider, J. Mannhart, and Yu. S. Barash, Appl. Phys. Lett. 86, 202505 (2005).

${ }^{6}$ D. Matthey, N. Reyren, J.-M. Triscone, and T. Schneider, Phys. Rev. Lett. 98, 057002 (2007).

${ }^{7}$ A. D. Caviglia, S. Gariglio, N. Reyren, D. Jaccard, T. Schneider, M. Gabay, S. Thiel, G. Hammerl, J. Mannhart and J.-M. Triscone, Nature 456, 624-627 (2008).

${ }^{8}$ C. H. Ahn, S. Gariglio, P. Paruch, T. Tybell, L. Antognazza, and J.-M. Triscone, Science 284, 1152-1155 (1999).

${ }^{9}$ S. Gariglio, C. H. Ahn, D. Matthey, and J.-M. Triscone, Phys. Rev. Lett. 88, 067002 (2002).

${ }^{10}$ K. Ueno, S. Nakamura, H. Shimotani, A. Ohtomo, N. Kimura, T. Nojima, H. Aoki, Y. Iwasa, and M. Kawasaki, Nature Mater. 7, 855-858 (2008).

${ }^{11}$ J. T. Ye, S. Inoue, K. Kobayashi, Y. Kasahara, H. T. Yuan, H. Shimotani, and Y. Iwasa, Nature Mater. 9, 125-128 (2010).

${ }^{12}$ A. S. Dhoot, S. C. Wimbush, T. Benseman, J. L. MacManus-Driscoll, J. R. Cooper, and R. H. Friend, Adv. Mater. 22, 2529-2533 (2010).

${ }^{13}$ H. Yuan, H. Shimotani, A. Tsukazaki, A. Ohtomo, M. Kawasaki, and Y. Iwasa, Adv. Funct. Mater. 19, 1046-1053 (2009).

${ }^{14}$ S. G. Haupt, D. R. Riley, J. Zhao, J.-P. Zhou, J. H. Grassi, and J. T. McDevitt, Proc. SPIE 2158, 238-249 (1994).

${ }^{15}$ A. Gozar, G. Logvenov, L. F. Kourkoutis, A. T. Bollinger, L. A. Giannuzzi, D. A. Muller, and I. Bozovic, Nature 455, 782-785 (2008).

${ }^{16}$ G. Logvenov, A. Gozar, and I. Bozovic, Science 326, 699-702 (2009).

${ }^{17}$ S. Smadici, J. C. T. Lee, S. Wang, P. Abbamonte, G. Logvenov, A. Gozar, C. Deville Cavellin, and I. Bozovic, Phys. Rev. Lett. 102, 107004 (2009).

${ }^{18}$ I. Bozovic, IEEE Trans. Appl. Supercond. 11, 2686-2695 (2001).

${ }^{19}$ K. Ueno, S. Nakamura, H. Shimotani, H. T. Yuan, N. Kimura, T. Nojima, H. Aoki, Y. Iwasa, and M. Kawasaki, Nat. Nanotechnol. 6, 408-412 (2011).

${ }^{20}$ S. Zhang, N. Sun, X. He, X. Lu, and X. Zhang, J. Phys. Chem. Ref. Data 35, 1475-1517 (2006). 